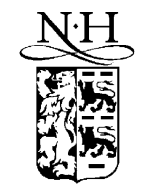

ELSEVIER
Applied Surface Science 168 (2000) 186-193

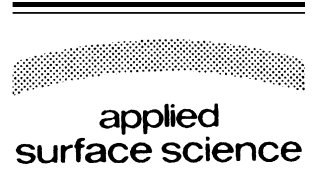

$\overline{\overline{\text { www.elsevier.nl/locate/apsusc }}}$

\title{
Materials modification by electronic excitation
}

\author{
A.M. Stoneham ${ }^{\mathrm{a}, *}$, Noriaki Itoh $^{\mathrm{b}}$ \\ ${ }^{a}$ Centre for Materials Research, Department of Physics and Astronomy, University College London, \\ London WC1E 6BT, UK \\ b1-7-35 Uenohigashi, Toyonaka, Osaka 560-0013, Japan
}

\begin{abstract}
Electronic excitation by lasers or electron beams can modify the properties of materials. The changes are not just due to heat, nor do they result from the well-known collision dynamics of much radiation damage. Everyday examples of modification by electronic excitation include photography, and photochromics (such as sunglasses) which change colour. In the last few years it has become clear that excitation can offer novel types of modification, with better-controlled changes. The field has evolved through a mix of basic science, of new laser and electron beam tools, and of new needs from microelectronics, photonics and nanotechnology. Underlying this development are some common themes which integrate the basic science and its applications. These include especially the ideas of energy localisation and charge localisation. There are detailed comparisons of experiment and theory for halides, but there is a wealth of information for other materials. From this, we identify ways to connect understanding to technological needs, like selective removal of material, controlled changes, altering the balance between process steps, and possibilities of quantum control. The field is reviewed in full in our recent book [N. Itoh, A.M. Stoneham, Materials Modification by Electronic Excitation, Cambridge University Press, Cambridge, 2000]. (C) 2000 Elsevier Science B.V. All rights reserved.
\end{abstract}

Keywords: Excited states; Colour change; Adhesion; Writing gratings; Surface modification

\section{Introduction}

Photography gives a very old example of changing a material by exciting it with light. Ideas for replacing dental drills by laser treatment, and photochromic sunglasses are other examples. These are special cases of a more general approach, which uses lasers or electron beams to tackle new needs, especially in the new technologies of microelectronics, photonics (lasers and optical fibres) and nanotechnology. In the last few years, new approaches have evolved which promise unique control and low thermal budgets. These methods exploit ideas and understanding from basic science, such as energy localisation and charge

* Corresponding author. localisation. Controlled excitation ("taming the excited state") has the potential to meet technological needs, like selective removal of material, controlled changes in selected regions, altering the balance between process steps, and possibilities of quantum control.

Most areas of major practical importance fall into one of six categories: (a) Removal of surface material, especially in a controlled way; (b) Modification of surface layers; (c) Modification of the bulk in a selected way; (d) Altering the absolute rate of a bulk or surface process; (e) Switching from one process to another: altering branching ratios. In this category, we might also include the manipulation of excitation though materials design, even though the material itself is not modified by this excitation (e.g. the xerographic process relies on the manipulation of 
electron and hole excitations in Se). (f) The creation and control of damage (intentional or unintentional). Damage avoidance means minimising excitation processes which causes harmful modifications, or by arranging for damage to occur only in unimportant regions.

The key processes are often described in terms of "energy surfaces", the adiabatic total energy $E_{\mathrm{i}}(\boldsymbol{Q})$ for electronic state and nuclear configuration $\boldsymbol{Q}$. Often, we can concentrate on a single reaction coordinate (which is usually not a dynamically-independent normal mode), shown on the well-known configuration coordinate diagrams. A typical schematic form shows a parabolic energy surface $E_{\mathrm{i}}(\boldsymbol{Q})=E_{0 \mathrm{i}}+K(Q-$ $\left.Q_{0 \mathrm{i}}\right)^{2} / 2$, with the second term corresponding to the strain energy associated with displacement from the equilibrium displacement $Q_{0 \mathrm{i}}$. Critical issues concern (i) how rapidly energy is lost from coherent motion in $Q$, relative to the period of harmonic motion in $Q$, (ii) how rapidly there are transitions to other electronic states, whether radiative or non-radiative, and (iii) how the system branches between two such energy surfaces when they intersect. Much of the recent progress in controlled materials modification has been based on understanding the energy surfaces of the significant excited states.

\section{Classifying excitation}

In what manner does excitation cause change? There are four broad categories:

1. Excitation into selected electronic states. These selected states have very specific properties, exploiting special properties of the material. In sophisticated applications, the photon phase may prove critical. This is a standard idea in nuclear magnetic resonance, or in laser methods which exploit coherence. Such applications are not yet at all fully exploited in materials modification, but there are situations where there is promise. Some approaches exploit quantum interference between two continuous wave excitations [2] to alter the balance between two different sets of products on excitation. Related are those processes in which a laser driving one transition can "turn off" absorption associated with another transition (e.g. [3]). Then there are approaches which exploit two suitably-phased ultrafast laser pulses to control the decay channel in an excited state [4,5] The first pulse raises the system to energy surface $\mathrm{A}$, and the system cools through a crossing of two energy surfaces, A and B. The second pulse (which can be chirped from the first pulse) transfers population from the unwanted energy surface (A, say) to the desired surface (B, say). The idea is to reinforce the fraction in a chosen state after cooling through the intersection. Another class of process exploits the coherent phonons produced by light pulses (e.g. [6]), perhaps so that phonon coherence is exploited to control the flow of vibrational energy usefully. There will be mixed cases, in which both photons and phonons have roles. Finally, one should be aware of systems being considered for quantum computing, since they may have applications in modification. Such systems include bistable systems, for which one control parameter (essentially the barrier height) modifies dramatically the ease with which a move can be made from one minimum to another [7]. Potentially, there are many ways to take advantage of phase selectivity as well as spectroscopic selectivity. These include methods analogous to those used to manipulate spin states in nuclear magnetic resonance, and can achieve coherent population transfer among quantum states (e.g. [8]).

2. Energy input into a small but mesoscopic zone. In special cases, photon phase matters, as in writing an interference pattern in an optical fibre. The energy generates an excited state, often free carriers, electrons and holes, which recombine to produce local heating. This localised heat input helps to keep the process within a thermal budget. As microelectronic devices dimensions fall, such budgets are more important, yet the effects of defects become larger. The situation is especially demanding when defect production is a natural result of processing, as in ion implantation. The approximate validity of the " +1 " model helps the thermal budget, in that most $\mathrm{Si}$ vacancies appear to recombine with interstitials, leaving substitutional dopants and Si interstitials. Electronic excitation can enhance diffusion processes involved, so that the thermal budget is reduced 
even more. A major role of Rapid Thermal Processing (RTP, using optical methods instead of a thermal furnace) is thermal budget reduction. Photonics is also demanding materials modification of chosen small regions, so as to produce refractive index changes on length scales of optical wavelengths. One example relates to microfabrication for optical data storage, where two-photon polymerisation initiators have been proposed for three-dimensional data storage [9] The creation of local refractive index changes could be used to control the strength of coupling to an optical field, hence to ensure emission of a particular polarisation, or even to make local laser action possible [10]. Class II processes can generate significant local electric fields. Charge redistribution is especially important for X-rays or gammas, or when an electron beam is used. With the continuing drive to ultimate miniaturisation, issues of electronic excitation make contact with nanotechnology, i.e. the excitation of features consisting of very small numbers of atoms. There is little experimental work on such effects of excitation. Clearly (as in the photographic process) there will be special issues of defect stability and defect processes in systems of only a few hundred atoms (as in quantum dots which show optical shifts from confinement) or of a few tens of thousands of atoms (which show the Coulomb blockade). A single defect process can cause major changes. Electric fields are especially important: the loss of one charged ion creates a field large enough to affect electron hole-recombination dramatically.

3. Energy input to selected species from an ion beam. The selectivity is controlled either by the energy transfer (depending mainly on the target atom mass) or by a nuclear reaction (as in transmutation doping). In straightforward versions of ion implantation, the important features are not directly associated with electronic excitation.

4. Excitation of a gas phase species, with substrate heating. Excitation and heating can be confined to chosen regions. In photolytic deposition a chemical process in the gas phase is stimulated, and the reactive species modify the cold substrate. In processing III-Vs, metalorganics like trimethyl gallium are photolysed. In pyrolytic deposition, substrate heating provokes thermally-driven reactions with an ambient gas. In diamond film synthesis, it is likely that electronic excitation is important, even though this is not a part of any standard model.

\section{Underlying processes}

The underlying solid-state processes of materials modification are energy localisation, charge localisation, energy transfer, energy storage, and charge transfer. In halides and oxides, energy localisation is often associated with self-trapping (e.g. the exciton in quartz). Self-trapping can be a crucial component of charge localisation and transport (e.g. small polarons in halide phosphors). Core electron excitation and valence excitation can have very different consequences (e.g. the processes leading to ion emission from the $\mathrm{KCl}$ surface), which offers one route to control. Energy transfer can be from one group of lattice vibrations to another, rather than exchange between electrons and vibrational motion. For specific electronic states, cooling transitions are the means by which equilibrium recovers from excess local vibrations. Even for a chosen system, there can be a large variety of processes, as shown below. What is still extremely hard is to understand the selectivity of the processes, and how branching ratios are determined.

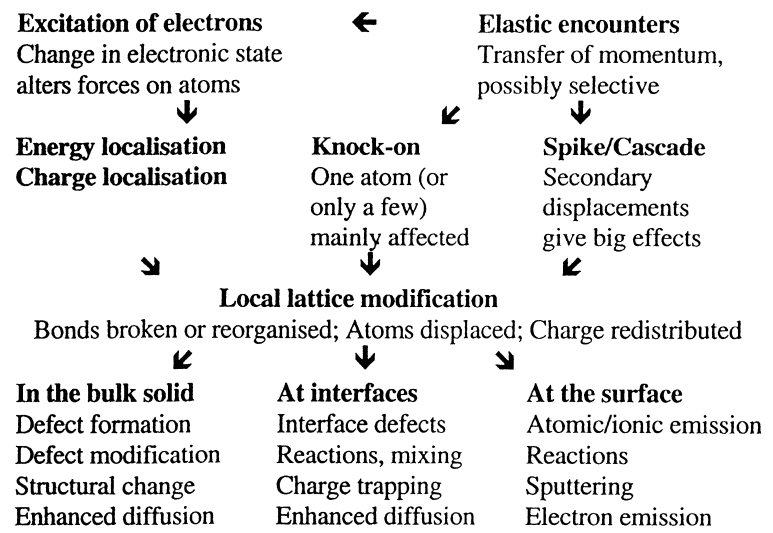

\section{Examples of materials modification}

Electronic excitation can be damaging as well as beneficial. Understanding gives the opportunity to 
design so as to minimise degradation, and thus to exploit the benefits. What are common features of damage processes? First, accelerated ageing tests will mislead if the degradation process is different in operation under accelerated conditions. This is why it is important to understand metastable defects, or defects which can emerge by evolution during processing (like thermal donors in $\mathrm{Si}$ ), and recombinationenhanced diffusion. Secondly, high electric fields arise in many damage processes. Thirdly, interfaces are a rich source of problems, from their ability to act as defect or impurity sinks. As an example, in Rapid Thermal Annealing, there is evidence that $\mathrm{P}_{\mathrm{b}}$ centres are exposed at the $\mathrm{Si} / \mathrm{SiO}_{2}$ interface, as passivating hydrogens are removed [11]. Fourthly, the damage may be in a part of the system other than the active device. The early degradation of blue GaN lasers was caused in part by ultraviolet light degrading plastic packaging [12].

\subsection{Writing in the bulk}

Photography is not just the domain of the domestic camera. Cosmic ray emulsions were used to identify elementary particles. The writing of features like gratings in optical fibres and optical storage media is arguably similar. In optical storage devices, the issues include contrast, information density, read rate, write rate, the need for refreshing the data, fatigue, and other limits on the number of read-write cycles. There are questions of how best to organise the stored image, and whether or not to use a hologram. Devices based on a defect creation mechanism seem to suffer from fatigue, and only suit situations which do not need frequent write operations.

In traditional silver halide photography, and indeed in several of the newer image storage devices for use with X-ray systems, there is a well-defined energy localisation step, although there is still debate about some of the precise mechanism. In the alkali halides, the position is clearer. Charge localisation occurs for holes, with the self-trapped hole $\left(\mathrm{V}_{\mathrm{k}}\right.$ centre $)$ essentially a halogen molecular ion, $\mathrm{X}_{2}{ }^{-}$. Energy localisation involves an electron-hole complex which, in some cases, is the obvious $\left(\mathrm{V}_{\mathrm{k}}, \mathrm{e}\right)$ on-centre exciton. However, in the ground state of the exciton (and there are many accessible excited states, including those with electron excited, hole excited, both excited, and spin triplet states) there are strong forces tending to drive the $\mathrm{X}_{2}{ }^{-}$molecular ion along the close-packed $\left(\begin{array}{lll}1 & 1 & 0\end{array}\right)$ axis. A part of this driving force comes from the energy gain when the electron is trapped in the anion vacancy left as the molecular ion moves. There is, therefore, a mechanism (in many, but not all, alkali halides) by which optical excitation can create defect pairs. For the alkali halides, the defect pairs comprise an $\mathrm{F}$ centre (neutral anion vacancy) and an $\mathrm{H}$ centre (neutral halogen interstitial). At the very least, these processes require that the exciton energy exceeds the energy to create an $\mathrm{F}-\mathrm{H}$ pair. This condition holds for many halides, but is marginal for many oxides (especially closed-shell oxides like $\mathrm{MgO}$ or $\mathrm{Al}_{2} \mathrm{O}_{3}$ ) and fails for semiconductors. In semiconductors, one is more likely to find metastable states, enhanced diffusion, or surface effects. Subsequent processes, on prolonged excitation, involve mechanisms to convert point defects into dislocation structures. Since the initial defects are often on a single sublattice, the means to create dislocations involving both sublattices is important. In alkali halides, the interstitial halogens "punch out" anion-cation divacancies, into which the halogen molecule goes. In III-V semiconductors, probably an antisite defect is created.

Self-trapped excitons are formed in $\alpha$-quartz; in essence, the hole is concentrated on a displaced $\mathrm{O}$ and the electron on a neighbouring $\mathrm{Si}$, rather like an incipient anion vacancy-interstitial pair. Oxygen defects are formed with low efficiency $[1,13]$. Vitreous silica is used widely for lithographic optics. Its large band gap, ease of manufacture (including the successful removal of most $\mathrm{Al}$ and $\mathrm{H}$ ), and years of experience give it major advantages. Miniaturisation in microelectronics is driving moves to higher-energy photons (193 nm lithography for $130 \mathrm{~nm}$ technology is scheduled for 2003), which are more able to cause deterioration. Ideally, coatings for projection optics should last 10 years without degradation $\left(10^{11}\right.$ pulses, each of $1 \mathrm{~mJ} / \mathrm{cm}^{2}$ ). Trials in 1998 include reported successful coatings exposed to $10^{9}$ pulses of $193 \mathrm{~nm}$ light at $15 \mathrm{~mJ} / \mathrm{cm}^{2}$. Compaction, a slow and slight change in dimensions as the optical system is used over periods of months or years, is driving moves to crystalline materials, like $\mathrm{CaF}_{2}$. For crystalline materials, however, dislocations are a problem, partly because of birefringence, and partly because they may nucleate damage. Compaction may be associated 
with point defects or impurities, or with altered bonding patterns and changes in network topology. In optical fibres, these processes can be useful, since they allow gratings to be written. It is an aim of much present work to create photonic features, like gratings, which give substantial contrast in refractive index and which remain stable. Such features allow the processing of signals within an optical fibre, and open opportunities in areas as diverse as communications and sensing.

\subsection{Smoothing or roughening the surface}

Laser and electron excitation can smooth surfaces. Scanning probe microscopy (SPM) shows atom removal occurs preferentially near vacancies or surface steps, so that incomplete layers of atoms are removed before new vacancies form in deeper layers. Energy localisation is needed at special surface sites. The process is gentler than the (less-effective) quasimelting of laser annealing. Laser excitation can also adjust surface stresses, primarily through rendering defects or dislocations more mobile. There are still important questions about mechanism. When the bandgap energy is less than the energy to form a defect or to remove an atom from surfaces, the excitonic mechanism cannot be effective. The loss of an electron from a bonding orbital (excitation to an antibonding state) might be effective, but would need a lifetime of perhaps $100 \mathrm{fs}$.

SPM observations of the Si (1 111 1) $7 \times 7$ surface irradiated with $193 \mathrm{~nm}$ lasers [14] show vacancies in the $7 \times 7$ reconstructed structure generated without disturbing the remaining atoms. The number of removed atoms increased linearly with fluence (as for an excitonic mechanism), with cross-section $0.52 \times 10^{-6} \AA^{2}$ for the clean surfaces and $1.8 \times$ $10^{-6} \AA^{2}$ for chlorinated surfaces, independent of temperature from room temperature to $1000^{\circ} \mathrm{C}$. These cross sections are much lower than for the excitonic mechanism on surfaces. Later work $[15,16]$ showed that atoms are removed from surfaces of GaAs $\left(\begin{array}{lll}1 & 1 & 0\end{array}\right)$, Si $\left(\begin{array}{lll}1 & 1 & 0\end{array}\right) 2 \times 1$ and Si $\left(\begin{array}{lll}1 & 1 & 1\end{array}\right) 7 \times 7$ surfaces by 90 $2000 \mathrm{eV}$ electron irradiation. Again, yields were linear functions of fluence, with cross-sections $4.4 \times 10^{-4} \AA^{2}$ for $\operatorname{GaAs}\left(\begin{array}{lll}1 & 1 & 0\end{array}\right)$ and $1 \times 10^{-4} \AA^{2}$ for $\mathrm{Si}\left(\begin{array}{lll}1 & 1 & 1\end{array}\right)$ for $100 \mathrm{eV}$ electrons. Such electron energies are too low to create defects by elastic encounters. Since an electron of $100 \mathrm{eV}$ creates about 30 excitations in silicon, the average number of $\mathrm{e}-\mathrm{h}$ pairs per emitted atom is similar to that for laser irradiation. The SPM data show that vacancy creation by electrons occurs randomly, unrelated to existing defects, whereas surface defects enhance emissions under laser irradiation. For surfaces irradiated by lower-energy photons, yields superlinear in the fluence are seen by SPM. Ishikawa et al. [17] found vacancies created on the Si $\left(\begin{array}{lll}1 & 1 & 1\end{array}\right)$ $7 \times 7$ surface by irradiation with $2.48 \mathrm{eV}$ laser pulses. Kanasaki et al. used $1.7-3.0 \mathrm{eV}$ photons, finding that the removal rate is enhanced resonantly around $2.0 \mathrm{eV}$ [18]. The number of atoms emitted in the ground state is a superlinear function of fluence, and agrees with that of the number of vacancies generated. Electrons with energies of 3-10 eV create vacancies on the $\mathrm{Si}$ (1 111 1) $7 \times 7$ surface; those of $1.8 \mathrm{eV}$ do not [19]. The superlinear dependence is observed only for low energy laser pulses, for which excited states generated during a laser pulse can be excited further within the same pulse. The SPM data show that atoms neighbouring defect sites on surfaces are emitted in preference to those at perfect surface sites. $2.3 \mathrm{eV}$ laser pulses only create defects on surfaces of GaAs $\left(\begin{array}{lll}1 & 1 & 0\end{array}\right)$ if the surfaces are already damaged by electron irradiation [16]. For Si ( $\left.\begin{array}{llll}1 & 1 & 1\end{array}\right) 7 \times 7$ surface, the removal rate of atoms neighbouring a vacancy is $10^{2}$ higher than those on undamaged reconstructed surface [14]. A monolayer of $\mathrm{Si}\left(\begin{array}{lll}1 & 1 & 0\end{array}\right) 2 \times 1$ can be removed by $2.3 \mathrm{eV}$ laser irradiation leaving unreconstructed surfaces resistive to laser irradiation of the same fluence $[20,21]$. Defect-initiated atomic emission under laser irradiation has been studied extensively for surfaces both of silicon and compound semiconductors [22]. The yields of the emissions are superlinear functions of fluence, and the fluence range at which emissions start depends on defect type. The emission yield of atoms neighbouring vacancy clusters on GaAs $\left(\begin{array}{lll}1 & 1 & 0\end{array}\right)$ under electron irradiation is a factor of 5 larger than those on terraces [16].

\subsection{Changing colour, adhesion and passivation}

Sunglasses are the best-known photochromics. Darkening in strong light occurs in an oxide glass containing a suspension of silver-halide-enriched particles with traces of the cuprous ion, $\mathrm{Cu}^{+}$. The small particles, typically $10 \mathrm{~nm}$ across, do not scatter light 
significantly. Exposure to ultraviolet light creates $\mathrm{Cu}^{2+}$ and $\mathrm{Ag}$ atoms, which aggregate to form $\mathrm{Ag}$ colloids. Both the cupric ion and the colloids absorb in the visible. This non-equilibrium state persists whilst the light is strong, and recovers when the light is removed. This system responds to an external stimulus in a desirable way. The glass is a "smart" material, and one of the few cases which are justifiably smart. In many cases, the materials respond to an external stimulus too weakly to be useful, or there is a problem in finding something important to be smart about: many so-called smart materials are merely "alert", in that they provide a signal which allows some powered device to achieve the response. Sunglasses also show a system taken to a distinctly non-equilibrium state. Insulators can exist for long periods with charges out of equilibrium. There is the no-defined Fermi energy, since populations have not achieved a Fermi distribution. The non-equilibrium state can last for archaeological periods in the absence of light; indeed, this is the basis of some of the most important thermoluminescence methods of dating archaeological materials and authenticating art ceramics [23,24].

Optically-induced charge transfer reactions such as $\mathrm{A}^{\mathrm{o}}+\mathrm{B}^{\mathrm{o}} \rightarrow \mathrm{A}^{+}+\mathrm{B}^{-}$are common. Excitation changes species and redistributes charge. The redistribution can be over space, over species, or in more complicated forms. Some consequences are desirable, others not so. There may be a change of colour, giving photochromism. Many photochromic systems are surveyed by Amodei [25], who lists performance criteria and key property values. The inorganic systems are mainly based on colour centres or on transition metals. The organic systems are more varied. The main classes are cis-trans isomerization, a characteristic molecular restructuring; heterolytic cleavage, in which a covalent bond is broken to produce a pair of ions, one positively charged, the other negatively charged; homolytic cleavage, to produce a pair of radicals; tautomeric processes, in which an atom (often hydrogen) is transferred; and triplet-state formation. Colour changes which can be reversed optically provide options for information storage. This might be a change in colour or dichroism of a region associated with a charge transfer. The region will be at least similar in size to the optical wavelength. Ideally, recording, reading and erasing operations are done at different wavelengths. One recent proposal [26] is for a non-volatile holographic memory in $\mathrm{LiNbO}_{3}$ based on $\mathrm{Mn}(2+/ 3+)$ and $\mathrm{Fe}(2+/ 3+)$. Other variants exploit hole-burning; individual ions with narrow homogeneous widths are accessed in inhomogeneously broadened lines [26-29,30]. Optical memory technologies vary greatly in mechanism and in need addressed. Examples from an enormous literature include cation-deficient spinel ferrites for high-density, write-once, read many times systems [31] and stoichiometric $\mathrm{LiTaO}_{3}$ for near-UV dynamic holography [32], where electron transfer processes are involved.

Charge transfer can redistribute charge in two main ways. All cases involve redistribution over species or sites. In glasses which act as windows for spent nuclear fuel examination, there is redistribution over $\mathrm{Ce}$ in the glasses: the sites which are most stable for $3+$ ions lose an electron and sites stable as $4+$ gain an electron. Ce is special, in that neither the $3+$ nor the $4+$ state absorbs in the visible, so the presence of $\mathrm{Ce}$ combats darkening. For the same reason, Ce-based glasses are used to protect optical devices for use in space. The colour of many gemstones and glasses results from redistribution of charge [33]. Redistribution over space normally requires an anisotropic stimulus. In the windows of caves for the examination of spent nuclear fuels, there is a "Compton Wind", in which electrons are moved by Compton scatter down a $\gamma$ flux. Traps can immobilise electronic carriers to such an extent that ionic motion is the main contribution to eliminating fields. This is so for Ce-doped glasses, for instance, where alkali ions or protons are the most mobile charged species.

Metal-oxide adhesion has an important electrostatic component: the ions in the oxide polarise the metal. This "image potential" term [34] is affected by charge redistribution. The methods most studied are anodic bonding (Mallory bonding) and radiation-enhanced adhesion. In both cases, charged defects are created. Even simple optical excitation should assist adhesion if there are ions in the oxide which can change charge state [35], since different charge states are favoured by the unpolarisable vacuum (bare surface) and highly polarisable metal. Similar electrostatic prinicples are used, often without a basic understanding of the mechanisms, for non-misting windows or non-misting mirrors for dental use. The same basic ideas encom- 
Table 1

Examples of excitation-induced changes, classed by material

Halides Photography is the classic example. Other imaging systems, whether for medical applications, or particle detection, extend the role of halide-based image formation. Halide-based photography is challenged by electronic approaches, although not for all applications. Other key examples for halides concern lack of materials modification for light below their wide bandgaps. The use of $\mathrm{CaF}_{2}$ for lithography optics, of $\mathrm{MgF}_{2}$ for $\mathrm{UV}$ windows, or of fluoride glasses for optical fibres, relies on a understanding of damage processes to avoid problems.

Oxides Common applications involve charge transfer, such as a change in colour, or to avoid colour. The most evident uses are in amorphous oxides (including glasses), especially silicon dioxide. Both the thin film form (oxide on silicon) and the fibre form have crucial technological roles. For the thin films, one wishes to minimise changes under electrical stress. For the fibre, controlled changes to create gratings and the like are important. The resistance of oxides to modification is an asset in many applications, such as coatings, or fibres to transmit laser power.

Semiconductors Applications concern processing: enhancement, acceleration, and control, and range from smoothing surfaces to improved lithography. There are new options as moves to smaller features demand lower thermal budgets. Rapid thermal processing for oxidation, for silicide formation, and for dopant activation after implants, probably involves some enhanced diffusion. Electronic excitation, whether by photons or electrons, offers local control (local enhancement of reactions or processes such as oxidation) and other opportunities which are not yet obvious.

pass anodic bonding, some of the adhesion of tyres on ice, and optical poling in optic fibres.

\section{Conclusions}

Can the materials modification processes be fully understood in those systems for which most information is available? Usually, there are significant gaps, even for photography. To the technologist, understanding provides the basis for optimisation and improvement, and progress is possible with even partial understanding. To the scientist, technology poses questions which may need more interesting science than we have identified from ideal systems. In the area of defect processes, there are still many gaps in our knowledge. The fundamental question for a technology in business is "Will it help financially?" With a new class or processes, such as materials modification by excitation, opportunities may be taken up only slowly. Some cases will fail, except for niches, like laser annealing. Others may fail on cost, simply because a laser photon is more expensive than hoped. But costs and needs are changing. In microelectronics, the needs of ultimate miniaturisation, ultimate speed, and low power operation drive towards new materials and new processes with lower thermal budgets. Exploitation of photonics in communications, or the ideas of quantum computing, if ever realised, will be science-based, needing further processes and materials. Business opportunities may follow technical opportunities, just as the compact disc required the semiconductor laser. The costs of lasers continue to fall, and their energy range continues to rise. New light sources are on the horizon. Given this mix of growing demand and improving technical capability, understanding the physical phenomena is crucial. Only then will one be informed so as to grasp the opportunities; only then can one avoid problems (Table 1).

\section{Acknowledgements}

This work was supported in part by EPSRC, British Telecom, the Royal Society and Fujitsu European Centre for Information Technology.

\section{References}

[1] N. Itoh, A.M. Stoneham, Materials Modification by Electronic Excitation, Cambridge University Press, Cambridge, 2000.

[2] P. Brumer, M. Shapiro, Farad Soc. Disc. 82 (1986) 177.

[3] R. Moseley, M. Dunn, Physics World, 1995, p. 30.

[4] D.J. Tanner, S.A. Rice, Adv. Chem. Phys. 70 (1988) 441.

[5] C.J. Bardeen, J. Che, K.R. Wilson, V.V. Yakovlev, P. Cong, B. Kohler, J.L. Krause, M. Messina, J. Phys. Chem. 101 (1997) 3815.

[6] G.A. Garrett, T.F. Albrecht, J.F. Whittaker, R. Merlin, Phys. Rev. Lett. 77 (1996) 3661.

[7] R. Landauer, in: A.J.G. Hey (Ed.), Feynman and Computation: Exploring the Limits of Computers, Perseus Books, Reading, MA, 1999, p. 77. 
[8] K. Bergmann, H. Theuer, B.W. Shore, Rev. Mod. Phys. 70 (1998) 3

[9] B. Cumpston, et al. Nature 398 (1999) 51.

[10] J. Faist, et al. Nature 387 (1999) 777.

[11] P. Hurley, C. Leveugle, A. Mathewson, D. Doyle, S. Whiston, J. Prendergast, P. Lundgren 1998, in: S. Ashok (Ed.), Proceedings of MRS Symposium 510, Defect and Impurity Engineered Semiconductors and Devices II, 1998, p. 569.

[12] A. Mills, III-Vs Review citing D. Steigerwald, M. Osinski, 11 (1) (1998) p. 46.

[13] N. Itoh, A.M. Stoneham, K. Tanimura, in: R. Devine (Ed.), Structure and Imperfections in Amorphous and Crystalline Silicas, Wiley, New York, 2000.

[14] X.H. Chen, J.C. Polanyi, D. Rogers, Surf. Sci. 376 (1997) 77.

[15] K. Nakayama, J.H. Weaver, Phys. Rev. Lett. 82 (1999) 980.

[16] B.Y. Han, K. Nakayama, J. Weaver, Phys. Rev. B 60 (1999) 13846.

[17] K. Ishikawa, J. Kanasaki, Y. Nakai, N. Itoh, Surf. Sci. 349 (1996) L153.

[18] J. Kanasaki, T. Ishida, K. Ishikawa, N. Itoh, Phys. Rev. Lett. 80 (1997) 4080.

[19] B.C. Stripe, A.M.A. Razeai, W. Ho, Phys. Rev. B 79 (1997) 4397.

[20] J. Xu, S.H. Overbury, J.F. Wendelkin, Phys. Rev. B 53 (1995) R4245.

[21] J. Xu, S.H. Overbury, J.F. Wendelkin, J. Vac. Sci. Technol. B 14 (1996) 918.
[22] N. Itoh, J. Kanasaki, A. Okano, Y. Nakai, Ann. Rev. Mater Sci. 25 (1995) 97.

[23] S.W.S. McKeever, Thermoluminescence in Solids, Cambridge University Press, Cambridge, 1985.

[24] J. Zimmerman, J. Phys. C4 (1971) 3265.

[25] J.J. Amodei, in: R.J. Pressley (Ed.), Handbook of Lasers with Selected Data on Optical Technology, CRC Press, Cleveland, US, 1971, p. 533.

[26] K. Buse, A. Adibi, D. Psaltis, Nature 393 (1998) 665.

[27] G. Castro, D. Haarer, R.M Macfarlane, H.P. Trommsdorf, US Patent No. 4,101,976, 1978.

[28] W.E. Moerner, W. Lenth, G.C. Bjorklund, in: W.E. Moerner (Ed.), Topics in Current Physics 44, Springer, Berlin, 1988, p. 251.

[29] K. Holliday, in: S.G. Schulman (Ed.), U.P. Wild, of Molecular Luminescence Spectroscopy, Part 3, Wiley, New York, 1993, p. 149.

[30] A.M. Stoneham, Rev. Mod. Phys. 41 (1969).

[31] L. Bouet, P. Teilhades, I. Pasquet, C. Bonningue, S. LeBrun, A. Rousset, Jpn. J. Appl. Phys. (Part 1) 38 (1999) 1826.

[32] Y. Furukawa, K. Kitamura, K. Niwa, H. Hatano, P. Bernasconi, G. Montemezzani, P. Gunter, Jpn. J. Appl. Phys. (Part 1) 38 (1999) 1816.

[33] K. Nassau, The Physics and Chemistry of Color, Wiley, New York, 1983.

[34] D.M. Duffy, J.H. Harding, A.M. Stoneham, J. Appl. Phys. 76 (1995) 2791.

[35] D.M. Duffy, J.H. Harding, A.M. Stoneham, Acta Met. 43 (1995) 1559. 